\title{
Exact Penalization and Optimality Conditions for Approximate Directional Minima
}

\section{Teodor Chelmuş}

\begin{abstract}
In this paper, we study the concept of approximate directional efficiency for set-valued constrained and unconstrained optimization problems. In our work, we concerned with finding conditions under which the Clarke penalization technique can be applied, and we derive some optimality conditions via variational analysis tools such as limiting normal cones and its corresponding normal coderivative.
\end{abstract}

\section{Introduction}

This paper is devoted to combining several concepts and techniques in order to study some special kinds of efficiency in vector optimization. More precisely, we discuss about approximate directional efficiency in constrained and unconstrained problems from the point of view of penalization techniques and optimality conditions. In this sense, the present work is linked with important papers from the literature dedicated to the concept of approximate solution in vector optimization (see $[1,5,6,11,13,16$, and the references therein).

The novelty we propose here is related to the extension of the concept of directional Pareto minimality, introduced in [3], to the case of approximate directional solutions. Therefore the penalization results and the necessary optimality conditions we derive here, even if it follows some known methods of investigation (see, for instance, 2]), it brings some new technical issues that we have to overcome.

We detail the organization of the paper and in addition, we emphasize several aspects concerning the methods we employ in our work and we describe the obtained results. The first section is dedicated to some notations and preliminaries data that we use in the present paper. The second section starts with the presentation of an abstract optimization problem that we will study together with the classical concept of Pareto minimality. In [5], it was studied the concept of approximate minimizers for vector optimization problems with set-valued maps and some interesting results were obtained only by using a penalization technique and some tools from variational analysis. So, motivated by the

Received December 7, 2020; Accepted October 21, 2021.

Communicated by Jein-Shan Chen.

2020 Mathematics Subject Classification. 54C60, 46G05, 90C46, 90C29, 90C30.

Key words and phrases. multiobjective optimization, directional minimality, exact penalty, variational analysis, approximate minima. 
aforementioned paper, we start this second section with the adapted definition of approximate minima to the directional framework. Then we move our attention to finding some penalization results for the following constrained vector optimization problem

minimize $F(x)$ subject to $x \in A$,

where $F: X \rightrightarrows X$ is a set-valued map and $\emptyset \neq A \subset X$. Firstly, we consider $A$ to be an arbitrary nonempty closed set and we impose for $F$ to satisfy the Lipschitz-like property. Secondly, we choose $A$ to be represented by $\widetilde{G}^{-1}(0)$, where $\widetilde{G}$ is an epigraphical type multifunction. This last case corresponds to the situation where the restrictions of the optimization problem are represented with the help of a set-valued map $\widetilde{G}$, and this naturally imposes some assumptions concerning the regularity property of the set-valued map $\widetilde{G}$. The third section is dedicated to the main result of this work which is based, in essence, on an incompatibility result. Since the objective set-valued map $F$ can't be directionally open around an approximate directional minimum point, using Mordukhovich coderivative, we derive a result that ensures the openness property of $F$. Then, based on a technical result and taking into account the contrapositive of the openness result mentioned above, we obtain a necessary condition for approximate minimizers. The end of this section is dedicated to an example that illustrates the main result.

The paper ends with some concluding remarks where we emphasize some possible future research ideas that could involve some computational approaches.

Throughout this paper, we assume that $X$ and $Y$ are normed vector spaces over the real field $\mathbb{R}$ and on a product of normed vector spaces we consider the sum norm, unless otherwise stated. By $B(x, \varepsilon)$ we denote the open ball with center $x$ and radius $\varepsilon>0$ and by $B_{X}$ the open unit ball of $X$. In the same manner, $D(x, \varepsilon)$ and $D_{X}$ denote the corresponding closed balls. The symbol $S_{X}$ stands for the unit sphere of $X$. By $X^{*}$ we denote the topological dual of $X$, while $w^{*}$ stands for the weak* topology on $X^{*}$.

Let $F: X \rightrightarrows Y$ be a set-valued map. As usual, the graph of $F$ is

$$
\operatorname{Gr} F:=\{(x, y) \in X \times Y \mid y \in F(x)\}
$$

and the inverse of $F$ is the set-valued map $F^{-1}: Y \rightrightarrows X$ given by $(y, x) \in \operatorname{Gr} F^{-1}$ if and only if $(x, y) \in \operatorname{Gr} F$. Consider a nonempty subset $A$ of $X$. Then the image of $A$ through $F$ is

$$
F(A):=\{y \in Y \mid \exists x \in A, y \in F(x)\}=\bigcup_{x \in A} F(x)
$$

and when $F:=f$ is a function, we will denote $f(X)$ by $\operatorname{Im} f$. The distance function associated to $A$ is $d_{A}: X \rightarrow \mathbb{R}$ given by

$$
d_{A}(x)=d(x, A):=\inf _{a \in A}\|x-a\|
$$


The topological interior, topological closure, the convex hull, and conic hull of $A$ are denoted, respectively, by $\operatorname{int} A, \operatorname{cl} A, \operatorname{conv} A, \operatorname{cone} A$. The negative polar of $A$ is

$$
A^{-}:=\left\{x^{*} \in X^{*} \mid x^{*}(a) \leq 0, \forall a \in A\right\}
$$

Let $K \subset X$ be a proper (that is, $K \neq\{0\}$ and $K \neq X$ ) convex and pointed cone. The positive dual cone of $K$ is

$$
K^{+}:=-K^{-}=\left\{x^{*} \in X^{*} \mid x^{*}(x) \geq 0, \forall x \in K\right\}
$$

and it is well-known that $K$ induces a partial order relation $\leq_{K}$ on $X$ by $x_{1} \leq_{K} x_{2}$ if and only if $x_{2}-x_{1} \in K$. If int $K \neq \emptyset$, in which case we say that $K$ is a solid cone, one can consider as well the strict partial order relation $<_{K}$ by $x_{1}<_{K} x_{2}$ if and only if $x_{2}-x_{1} \in \operatorname{int} K$.

We will deal with vectorial problems with set-valued objectives and for this, we consider a pointed convex cone $Q$ on $Y$ which, characterizes a (strict) partial order relation on $Y$. We denote by $S_{Q}$ the intersection between $Q$ and unit sphere from $Y$.

\section{Approximate minima and exact penalization}

Take a set-valued mapping $F: X \rightrightarrows Y$, and let us consider the following geometrically constrained optimization problem with set-valued objective

$$
\text { minimize } F(x) \text { subject to } x \in A \text {, }
$$

where $A \subset X$ is a closed nonempty set.

The minimality is understood in the vectorial or Pareto sense as follows.

A point $(\bar{x}, \bar{y}) \in \operatorname{Gr} F \cap(A \times Y)$ is a local Pareto minimum point for $F$ on $A$ if there exists a neighborhood $U$ of $\bar{x}$ such that $\bar{x}$ is a Pareto minimum for $F(U \cap A)$, that is

$$
(F(U \cap A)-\bar{y}) \cap-Q=\{0\} .
$$

Suppose that $Q$ is a solid cone. Similarly, the point $(\bar{x}, \bar{y}) \in \operatorname{Gr} F \cap(A \times Y)$ is a local weak Pareto minimum point for $F$ on $A$ if there exists a neighborhood $U$ of $\bar{x}$ such that $\bar{x}$ is a weak Pareto minimum of $F(U \cap A)$, that is

$$
(F(U \cap A)-\bar{y}) \cap-\operatorname{int} Q=\emptyset .
$$

The vectorial notions described by 2.1 and (2.2) cover as well the situation where $F:=f$ is a function (in which case $\bar{y}=f(\bar{x})$ will not be mentioned) and the situation of classical local minima in the scalar case (in which case we drop the label "Pareto"). 
Moreover, when $U=X$ we have the global minimality and we simply drop the word "local".

In what follows, considering the notation used for describing the problem $(\sqrt{\mathrm{P}})$, we present some approximate directional concepts of efficiency. Besides the proper pointed convex and solid cone $Q \subset Y$ we consider as well a nonempty closed set $L \subset S_{X}$.

Definition 2.1. Let $\delta, \varepsilon \in(0, \infty), c \in Q \backslash\{0\}, \emptyset \neq A \subset X, A$ closed, and $(\bar{x}, \bar{y}) \in$ $\operatorname{Gr} F \cap(A \times Y)$.

(i) $(\bar{x}, \bar{y})$ is said to be an $(\varepsilon, \delta, c)$-approximate Pareto directional minimum point for $F$ on $A$ with respect to $L$, and we denote $(\bar{x}, \bar{y}) \in(\varepsilon, \delta, c)-\operatorname{DirMin}(F, A, L, Q)$, if

$$
(F(B(\bar{x}, \varepsilon) \cap A \cap[\bar{x}+\text { cone } L])+\delta c-\bar{y}) \cap-Q=\emptyset .
$$

(ii) $(\bar{x}, \bar{y})$ is said to be an $(\varepsilon, \delta)$-approximate Pareto directional minimum point for $F$ on $A$ with respect to $L$, and we denote $(\bar{x}, \bar{y}) \in(\varepsilon, \delta)-\operatorname{DirMin}(F, A, L, Q)$, if

$$
\left(F(B(\bar{x}, \varepsilon) \cap A \cap[\bar{x}+\text { cone } L])+\delta S_{Q}-\bar{y}\right) \cap-Q=\emptyset .
$$

Suppose, in addition, that $Q$ is solid.

(iii) $(\bar{x}, \bar{y})$ is said to be an $(\varepsilon, \delta, c)$-approximate weak Pareto directional minimum point for $F$ on $A$ with respect to $L$, and we denote $(\bar{x}, \bar{y}) \in(\varepsilon, \delta, c)-\operatorname{WDirMin}(F, A, L, Q)$, if

$$
(F(B(\bar{x}, \varepsilon) \cap A \cap[\bar{x}+\text { cone } L])+\delta c-\bar{y}) \cap-\operatorname{int} Q=\emptyset .
$$

(iv) $(\bar{x}, \bar{y})$ is said to be an $(\varepsilon, \delta)$-approximate weak Pareto directional minimum point for $F$ on $A$ with respect to $L$, and we denote $(\bar{x}, \bar{y}) \in(\varepsilon, \delta)-\operatorname{WDirMin}(F, A, L, Q)$, if

$$
\left(F(B(\bar{x}, \varepsilon) \cap A \cap[\bar{x}+\text { cone } L])+\delta S_{Q}-\bar{y}\right) \cap-\operatorname{int} Q=\emptyset .
$$

The concepts defined by (i) and (iii) are directional generalizations of the local (weak) Pareto solutions (see [1]), while (ii) and (iv) are stronger version of (i) and (iii).

Remark 2.2. Consider the epigraphical set-valued map $\widetilde{F}: X \rightrightarrows Y$ be defined as $\widetilde{F}(x)=$ $F(x)+Q$. It can be shown that an approximate minimum point of $F$ (in the sense of each item in the above definition) is also an approximate minimum point for $\widetilde{F}$.

Example 2.3. For a better understanding, we illustrate the above definition by an interesting example. Let $\alpha>0$. Setting $X=A=\mathbb{R}, Y=\mathbb{R}^{2}, Q=\mathbb{R}_{+}^{2}, L=\{+1\}$, we define the set-valued map $F: X \rightrightarrows Y$ by

$$
F(x):= \begin{cases}\{x\} \times\{0\} & \text { if } x \in[-\alpha, 0], \\ \{x\} \times\left[-\sqrt{1-(x-1)^{2}}, \sqrt{1-(x-1)^{2}}\right] & \text { if } x \in(0,2], \\ \mathbb{R}^{2} & \text { if } x \in[-\alpha, 2]^{c} .\end{cases}
$$


Fix $\varepsilon>0, \delta>0$, and $c \in S_{\mathbb{R}_{+}^{2}}$. Note that if $c \in S_{\mathbb{R}_{+}^{2}} \backslash\{(1,0)\}$, then $(\bar{x},(\bar{x}, 0))$, for every $\bar{x} \in[-\alpha, 0]$, is an $(\varepsilon, \delta, c)$-approximate directional minimum point for $F$ on $A$ with respect to $L$. Note that these points are also $(\varepsilon, \delta, c)$-approximate weak directional minima.

Take $c=(1,0)$ and $(\bar{x}, \bar{y}) \in \operatorname{Gr} F$ with $\bar{x} \in[-\alpha, 0]$ and $\bar{y}=(\bar{x}, 0)$.

Let us consider two cases:

- if $\delta>\alpha$, then $-\alpha+\delta>0$ and

$$
(F(B(\bar{x}, \varepsilon) \cap A \cap[\bar{x}+\text { cone } L])+\delta c-\bar{y}) \cap-Q=\emptyset .
$$

So, $(\bar{x},(\bar{x}, 0))$ is an $(\varepsilon, \delta, c)$-approximate directional minimum point for $F$ on $A$ with respect to $L$.

- if $\delta<\alpha$, then $-\alpha+\delta<0$ and

$$
(F(B(\bar{x}, \varepsilon) \cap A \cap[\bar{x}+\text { cone } L])+\delta c-\bar{y}) \cap-\operatorname{int} Q=\emptyset .
$$

In addition, the relation 2.3 does not hold for $\bar{x} \in(-\alpha+\delta, 0)$. So, $(\bar{x},(\bar{x}, 0))$ is not an $(\varepsilon, \delta, c)$ approximate directional minimum point for $F$ on $A$ with respect to $L$, but it is an $(\varepsilon, \delta, c)$-approximate weak Pareto directional minimum point for $F$ on $A$ with respect to $L$.

Exact penalization of Clarke's type is a useful technique that leads us to a precise conclusion about necessary optimality conditions for scalar or vector optimization problems. Notice that the next results are relatively close to some results in [2,5, but it is important to make a careful tracking of the constants involved in the definitions of approximate minima (see Definition 2.1). Inspired by a penalization technique applied for vector optimization problems in [14], we obtain a first penalization result presented below.

Theorem 2.4. Let $\varepsilon, \delta, \ell \in(0, \infty), c \in S_{Q}$ and $(\bar{x}, \bar{y}) \in \operatorname{Gr} F \cap(A \times Y)$. Suppose that $(\bar{x}, \bar{y})$ is an $(\varepsilon, \delta)$-approximate Pareto directional minimum point for $F$ on the closed set $A$ with respect to $L$ and that

$$
F\left(x^{\prime \prime}\right)+\ell\left\|x^{\prime \prime}-x^{\prime}\right\| c \subset F\left(x^{\prime}\right)+Q
$$

for all $x^{\prime}, x^{\prime \prime} \in B(\bar{x}, \varepsilon) \cap[\bar{x}+$ cone $L]$.

Then, for all $\ell^{\prime}>\ell$,

$$
(\bar{x}, \bar{y}) \in\left(\left(\ell+\ell^{\prime}\right)^{-1} \ell \varepsilon, \delta\right)-\operatorname{DirMin}\left(F_{0}, A, L, Q\right)
$$

where $F_{0}: X \rightrightarrows Y, F_{0}(x):=F(x)+\ell^{\prime} d(x, A \cap[\bar{x}+$ cone $L]) c$. 
Proof. Since $(\bar{x}, \bar{y})$ is an $(\varepsilon, \delta)$-approximate Pareto directional minimum point for the $F$ on $A$ with respect to $L$, then

$$
\left(F(B(\bar{x}, \varepsilon) \cap A \cap[\bar{x}+\text { cone } L])+\delta S_{Q}-\bar{y}\right) \cap-Q=\emptyset .
$$

Choose $\ell^{\prime}>\ell$ an arbitrary but fixed constant and $\rho=\ell \varepsilon\left(\ell+\ell^{\prime}\right)^{-1}$. Suppose to the contrary that $(\bar{x}, \bar{y})$ is not an $(\rho, \delta)$-approximate Pareto directional minimum point for the set-valued mapping $F_{0}$ on $X$ with respect to $L$. Whence there exist $x \in B(\bar{x}, \rho) \cap[\bar{x}+$ cone $L]$ and $y \in F(x)$ such that

$$
\bar{y}-y-\ell^{\prime} d(x, A \cap[\bar{x}+\text { cone } L]) c \in Q+\delta S_{Q}
$$

We consider separately the following two cases. Firstly if $d(x, A \cap[\bar{x}+$ cone $L])>0$, then

$$
d(x, A \cap[\bar{x}+\text { cone } L])<\frac{\ell^{\prime}}{\ell} d(x, A \cap[\bar{x}+\text { cone } L]),
$$

and, because the distance to a set is an infimum, it follows that there exists $u \in A \cap[\bar{x}+$ cone $L$ ] such that

$$
\|x-u\| \leq \frac{\ell^{\prime}}{\ell} d(x, A \cap[\bar{x}+\text { cone } L])
$$

Secondly if $d(x, A \cap[\bar{x}+$ cone $L])=0$, then $x \in \operatorname{cl}(A \cap[\bar{x}+$ cone $L])=A \cap[\bar{x}+$ cone $L]$ which implies that there exists $u:=x$ such that 2.6 is true. So,

$$
\begin{aligned}
\|u-\bar{x}\| & \leq\|u-x\|+\|x-\bar{x}\| \leq \frac{\ell^{\prime}}{\ell} d(x, A \cap[\bar{x}+\text { cone } L])+\|x-\bar{x}\| \\
& \leq\left(\frac{\ell^{\prime}}{\ell}+1\right)\|x-\bar{x}\|<\left(\frac{\ell^{\prime}}{\ell}+1\right) \rho=\varepsilon .
\end{aligned}
$$

Whence $u \in B(\bar{x}, \varepsilon) \cap A \cap[\bar{x} \cap$ cone $L]$. Returning to (2.4), we deduce that there exists $v \in F(u)$ such that

$$
y-v+l\|x-u\| c \in Q .
$$

Using the convexity of $Q$ and the relation (2.6), we obtain that

$$
y-v+\ell^{\prime} d(x, A \cap[\bar{x}+\text { cone } L]) c \in Q .
$$

Therefore, by adding the last relation and relation (2.5),

$$
\bar{y}-v \in\left(Q+\delta S_{Q}\right)+Q \subset Q+\delta S_{Q}
$$

This contradicts the minimality of $(\bar{x}, \bar{y})$. 
In what follows we present a penalization result for a generalized functional constraint optimization problem. Preparing the ground for the next result, we consider the admissible set $A$ to be of the following form

$$
G^{-1}(-Q)=\{x \in X \mid 0 \in G(x)+Q\}
$$

where $G: X \rightrightarrows Z$ is a multifunction and $Q \subset Z$ is a proper convex closed cone. We denote the problem $(\mathrm{P})$ with this type of constraint by $\left(\mathrm{P}_{G}\right)$. Observe that the set $G^{-1}(-Q)$ can be represented as $\widetilde{G}^{-1}(0)$, where $\widetilde{G}: X \rightrightarrows Z, \widetilde{G}(x)=G(x)+Q$ is the epigraphical setvalued map associated to $G$.

We will obtain a necessary condition for the problem $\left(\mathrm{P}_{G}\right)$ via penalization technique by appealing to the minimal time function (see [7] and the references therein) which is a useful tool for introducing a directional regularity notion for set-valued mappings. This approach was intensively used in 8 .

Consider $\emptyset \neq L \subset S_{X}$ and $\emptyset \neq \Omega \subset X$. The directional minimal time function with respect to $L$ is the application

$$
\begin{aligned}
\tau_{L}(x, \Omega) & :=\inf \{t \geq 0 \mid \exists u \in L \text { s.t. } x+t u \in \Omega\} \\
& =\inf \{t \geq 0 \mid(x+t L) \cap \Omega \neq \emptyset\} .
\end{aligned}
$$

In the particular case where $L=S_{X}$, then $\tau_{L}(\cdot, \Omega)=d(\cdot, \Omega)$. Moreover, we use the convention that $\tau_{L}(x, \emptyset)=+\infty$ for every $x \in X$ and we denote $\tau_{L}(x,\{u\})$ by $\tau_{L}(x, u)$. It is simple to check that one has

$$
\tau_{L}(x, u)<+\infty \quad \Longleftrightarrow \tau_{L}(x, u)=\|u-x\| \text { and } u-x \in \text { cone } L
$$

Let $F: X \rightrightarrows Y$ be a set-valued map and $(\bar{x}, \bar{y}) \in$ Gr F. In [4, Section 3.8, 3H], the authors underline the idea that the calmness property of the multifunction $F^{-1}$ is equivalent with the metric subregularity property of the multifunction $F$. In our setting, the corresponding definition of the two regularity properties for $F$ reads as follows: let $\varepsilon>0$ and $\ell>0$,

- $F$ is $(\varepsilon, \ell)$-directionally calm at $(\bar{x}, \bar{y})$ with respect to $L$ and $M$ if there exists a neighborhood $V$ of $\bar{y}$ such that, for every $x \in B(\bar{x}, \varepsilon)$,

$$
\sup _{y \in F(x) \cap V} \tau_{M}(y, F(\bar{x})) \leq \ell \tau_{L}(\bar{x}, x) .
$$

- $F$ is $(\varepsilon, \ell)$-directionally metric subregular at $(\bar{x}, \bar{y})$ with respect to $L$ and $M$ if for every $x \in B(\bar{x}, \varepsilon)$ we have

$$
\tau_{L}\left(x, F^{-1}(\bar{y})\right) \leq \ell \tau_{M}(\bar{y}, F(x))
$$


Remark 2.5. Note that the "one point" regularity properties defined above are particular cases of the notions of directional calmness and directional metric subregularity, respectively, defined and studied in [3]. Namely, the neighborhood of the element $\bar{x}$ is an open ball centered in $\bar{x}$ and of radius $\varepsilon$. For other similar concepts and interesting examples, we refer the reader to [8].

Remark 2.6. As already mentioned, it was shown in [3, Proposition 3.2] that the set-valued map $F$ is $(\varepsilon, \ell)$-directionally metric subregular at $(\bar{x}, \bar{y})$ with respect to $L$ and $M$ if and only if $F^{-1}$ is $(\varepsilon, \ell)$-directionally calm at $(\bar{y}, \bar{x})$ with respect to $M$ and $L$. Based on the properties of directional minimal time function, we can give a more convenient form of the directional calmness of the set-valued map $F^{-1}$. So, $F^{-1}$ is $(\varepsilon, \ell)$-directional calm at $(\bar{y}, \bar{x})$ with respect to $M$ and $L$ if, for every $\ell^{\prime}>\ell$ and $y \in B(\bar{y}, \varepsilon) \cap[\bar{y}+$ cone $M]$, we have

$$
F^{-1}(y) \cap B(\bar{x}, \varepsilon) \subset F^{-1}(\bar{y})-\left[0, \ell^{\prime}\|y-\bar{y}\|\right] \cdot L .
$$

This last inclusion will be used in order to describe a regularity property of the constraints set-valued map considered in the problem $\left(\mathrm{P}_{G}\right)$ and plays an important role in the next result inspired by a technique used in [15].

Theorem 2.7. Let $\varepsilon \in(0, \infty), \delta \in[0, \infty), \ell>0, m>0, m^{\prime}>m$ and $c \in S_{Q}$. Let $L \subset S_{X}$ be a nonempty closed set such that cone $L$ is convex and $(\bar{x}, \bar{y}) \in \operatorname{Gr} F \cap\left(G^{-1}(-Q) \times Y\right)$. Suppose that

(i) $(\bar{x}, \bar{y})$ is an $(\varepsilon, \delta)$-approximate directional Pareto minimum point for problem $\left(\mathrm{P}_{G}\right)$;

(ii) for every $x^{\prime}, x^{\prime \prime} \in B(\bar{x}, \varepsilon) \cap[\bar{x}+$ cone $L]$,

$$
F\left(x^{\prime \prime}\right)+\ell\left\|x^{\prime \prime}-x^{\prime}\right\| c \subset F\left(x^{\prime}\right)+Q
$$

(iii) $\widetilde{G}$ is $\left(2^{-1} \varepsilon, m\right)$-directionally metric subregular at $(\bar{x}, 0)$ with respect to $S_{Q}$ and $L$.

Denote $\lambda:=\max \left\{\ell, \ell m^{\prime}\right\}$. Then, for any $\lambda^{\prime}>\lambda$,

$$
((\bar{x}, 0), \bar{y}) \in\left(\left(\lambda+\lambda^{\prime}\right)^{-1} \lambda \varepsilon, \delta\right)-\operatorname{DirMin}\left(H_{0}, A, L, Q\right),
$$

where $H_{0}: X \times Z \rightrightarrows Y$ is defined by

$$
H_{0}(x, z):=F(x)+\ell m^{\prime}\|z\| c+\lambda^{\prime} d((x, z), \operatorname{Gr} \widetilde{G} \cap([\bar{x}+\text { cone } L] \times Q)) c .
$$

Proof. Fix $\rho=\min \left\{2^{-1},\left(2^{-1} m^{\prime}\right) \varepsilon\right\}$ and consider the set-valued map $H: X \times Z \rightrightarrows Y$,

$$
H(x, z):=F(x)+l m^{\prime}\|z\| .
$$

We will prove, firstly, that $((\bar{x}, 0), \bar{y})$ is an $(\rho, \delta)$-approximate directional Pareto minimum point for $H$ on $\operatorname{Gr} \widetilde{G}$ with respect to $L$, and, secondly, that $H$ has the Lipschitz-like 
property on $B((\bar{x}, 0), \varepsilon)$. The conclusion above will follow by applying Theorem 2.4 for the set-valued map $H$.

Suppose by contradiction that the point $((\bar{x}, 0), \bar{y})$ is not an approximate directional minimum point for $H$. Then there exist $(x, z) \in \operatorname{Gr} \widetilde{G} \cap B((\bar{x}, 0), \rho)$ with $x \in \bar{x}+$ cone $L$, $z \in 0+$ cone $S_{Q}=Q$, and $y \in F(x)$ such that

$$
\bar{y}-y+l m^{\prime}\|z\| c \in Q+\delta S_{Q} .
$$

Next, using the regularity property of $\widetilde{G}$ and Remark 2.6. we note that

$$
x \in \widetilde{G}^{-1}(z) \cap B\left(\bar{x}, 2^{-1} \varepsilon\right) \subset \widetilde{G}^{-1}(0)-\left[0, m^{\prime}\|z\|\right] \cdot L .
$$

Whence there exists $x^{\prime} \in \widetilde{G}^{-1}(0)=G^{-1}(-Q)$ such that

$$
x^{\prime}-x \in\left[0, m^{\prime}\|z\|\right] \cdot L \quad \text { and } \quad\left\|x^{\prime}-x\right\| \leq m^{\prime}\|z\|<2^{-1} \varepsilon .
$$

Since $x^{\prime} \in x+\left[0, m^{\prime}\|z\|\right] \cdot L$ and $x \in \bar{x}+$ cone $L$, it follows that $x^{\prime} \in \bar{x}+$ cone $L$, and since $x \in B\left(\bar{x}, 2^{-1} \varepsilon\right)$ and $\left\|x^{\prime}-x\right\| \leq 2^{-1} \varepsilon$, it follows that $x^{\prime} \in B(\bar{x}, \varepsilon)$.

So $x^{\prime}$ is a feasible point which belongs to the set $B(\bar{x}, \varepsilon) \cap[\bar{x}+$ cone $L]$. Applying the hypothesis (ii) for the pair $\left(x, x^{\prime}\right)$ we obtain the existence of an $y^{\prime} \in F\left(x^{\prime}\right)$ such that

$$
y-y^{\prime}+l\left\|x-x^{\prime}\right\| c \in Q .
$$

Using the fact that $c \in S_{Q}$ and the right-hand side of the relation (2.8), we have that

$$
y-y^{\prime}+l m^{\prime}\|z\| c \in Q .
$$

Adding the last inclusion and the one from the relation (2.7), we obtain that $\bar{y}-y \in Q$ which contradicts the minimality of the point $(\bar{x}, \bar{y})$.

Finally, choosing $x^{\prime}, x^{\prime \prime} \in B(\bar{x}, \varepsilon) \cap[\bar{x}+$ cone $L]$ and $z^{\prime} \in \widetilde{G}\left(x^{\prime}\right), z^{\prime \prime} \in G\left(x^{\prime \prime}\right)$, one can easily deduce from the Lipschitz-like property of the set-valued map $F$ that the set-valued map $H$ has the same property on the set $B((\bar{x}, 0), \varepsilon)$.

\section{Optimality conditions for approximate minima}

We start this section with an incompatibility result that point out the fact that the classical notions of linear openness (together with metric regularity or Aubin property) don't make any distinction between minima and maxima.

Theorem 3.1. Let $\varepsilon \in(0, \infty), \delta \in[0, \infty)$ and $c \in S_{Q}$. If $(\bar{x}, \bar{y}) \in \operatorname{Gr} F$ is an $(\varepsilon, \delta, c)$ approximate directional Pareto minimum point for the problem

$$
\min F(x), \quad x \in X,
$$

then one cannot have a positive $\delta^{\prime}>\delta$ such that

$$
B\left(\bar{y}, \delta^{\prime}\right) \cap[\bar{y}-\text { cone }\{c\}] \subset \widetilde{F}(B(\bar{x}, \varepsilon) \cap[\bar{x}+\text { cone } L]) .
$$


Proof. Suppose that $(\bar{x}, \bar{y}) \in \operatorname{Gr} F$ is an $(\varepsilon, \delta, c)$-approximate directional minimum point for $F$ with respect to $L$, then, using Remark 2.2 , $(\bar{x}, \bar{y})$ is an $(\varepsilon, \delta, c)$-approximate directional minimum point for $\widetilde{F}$ with respect to $L$, i.e.,

$$
\widetilde{F}(B(\bar{x}, \varepsilon) \cap[\bar{x}+\text { cone } L]) \cap(-Q-\delta c+\bar{y})=\emptyset,
$$

whence

$$
\widetilde{F}(B(\bar{x}, \varepsilon) \cap[\bar{x}+\text { cone } L]) \subset Y \backslash(-Q-\delta c+\bar{y}) .
$$

If, to the contrary, we have a positive $\delta^{\prime}>\delta$ such that

$$
B\left(\bar{y}, \delta^{\prime}\right) \cap[\bar{y}-\text { cone }\{c\}] \subset \widetilde{F}(B(\bar{x}, \varepsilon) \cap[\bar{x}+\text { cone } L]),
$$

then

$$
B\left(\bar{y}, \delta^{\prime}\right) \cap[\bar{y}-\text { cone }\{c\}] \subset Y \backslash(-Q-\delta c+\bar{y}),
$$

which implies the inclusion

$$
B\left(0, \delta^{\prime}\right) \cap[-\operatorname{cone}\{c\}] \subset Y \backslash(-Q-\delta c)
$$

But then the element $-\delta c \in B\left(0, \delta^{\prime}\right) \cap[-\operatorname{cone}\{c\}]$, a contradiction.

While it cannot be possible to have a directional openness at an approximate efficiency point, after making a careful choice of some constants, we focus on proving that $F$ can be directional open at an arbitrary point $(\bar{x}, \bar{y}) \in \operatorname{Gr} F$. The Theorem 3.10 from [9] is the key to the proof of the next result. Before moving forward, we will briefly recall some construction introduced by Mordukhovich and his collaborators (see 12$]$ ).

Consider $\Omega$ a nonempty subset of $X, x \in \Omega$ and $\varepsilon \geq 0$. The set of $\varepsilon$-normals to $\Omega$ at $x$ is

$$
\widehat{N}_{\varepsilon}(\Omega, x):=\left\{\begin{array}{l|l}
x^{*} \in X^{*} & \limsup _{u \rightarrow x} \frac{\left\langle x^{*}, u-x\right\rangle}{\|u-x\|} \leq \varepsilon
\end{array}\right\},
$$

where $u \stackrel{\Omega}{\rightarrow} x$ means that $u \rightarrow x$ and $u \in \Omega$. If $\varepsilon=0$, the elements in the right-hand side of (3.1) are called Fréchet normals and their collection, denoted by $\widehat{N}(\Omega, x)$, is the Fréchet normal cone to $\Omega$ at $x$.

Let $\bar{x} \in \Omega$. The basic (or limiting/Mordukhovich) normal cone to $\Omega$ at $\bar{x}$ is

$$
N(\Omega, \bar{x}):=\left\{x^{*} \in X^{*} \mid \exists \varepsilon_{n} \downarrow 0, x_{n} \stackrel{\Omega}{\rightarrow} \bar{x}, x_{n}^{*} \stackrel{w^{*}}{\rightarrow} x^{*}, x_{n}^{*} \in \widehat{N}_{\varepsilon_{n}}\left(\Omega, x_{n}\right), \forall n \in \mathbb{N}\right\} .
$$

In particular, if $\Omega:=K$ is convex, then

$$
N(K, \bar{x})=\widehat{N}(K, \bar{x})=\left\{x^{*} \in X^{*} \mid\left\langle x^{*}, k-\bar{x}\right\rangle \leq 0, \forall k \in K\right\} .
$$


In the following, we present two concepts of coderivatives for set-valued maps. Let $F: X \rightrightarrows Y$ be a set-valued map and $(\bar{x}, \bar{y}) \in \mathrm{Gr} F$. Then the Fréchet coderivative of $F$ at $(\bar{x}, \bar{y})$ is the set-valued map $\widehat{D}^{*} F(\bar{x}, \bar{y}): Y^{*} \rightrightarrows X^{*}$ given by

$$
\widehat{D}^{*} F(\bar{x}, \bar{y})\left(y^{*}\right):=\left\{x^{*} \in X^{*} \mid\left(x^{*},-y^{*}\right) \in \widehat{N}(\operatorname{Gr} F,(\bar{x}, \bar{y}))\right\} .
$$

Similarly, the normal coderivative of $F$ at $(\bar{x}, \bar{y})$ is the set-valued map $D^{*} F(\bar{x}, \bar{y}): Y^{*} \rightrightarrows X^{*}$ given by

$$
D^{*} F(\bar{x}, \bar{y})\left(y^{*}\right):=\left\{x^{*} \in X^{*} \mid\left(x^{*},-y^{*}\right) \in N(\operatorname{Gr} F,(\bar{x}, \bar{y}))\right\} .
$$

Let $\sigma>0$. We recall that $F$ has the Aubin property on $B(\bar{x}, \sigma) \times B(\bar{y}, \sigma)$ if there exists $\kappa \geq 0$ such that

$$
F\left(y^{\prime}\right) \cap B(\bar{x}, \sigma) \subset F(y)+\kappa\left\|y^{\prime}-y\right\| D_{Y}
$$

for every $y^{\prime}, y \in B(\bar{y}, \sigma)$.

We will give now an ancillary result that helps us in the proof of the main result of this paper.

Lemma 3.2. Consider $\psi, \varphi:[0, \infty) \rightarrow[0,1)$,

$$
\psi(x):=(1+x)^{-1 / 2}-(1+x)^{-1}, \quad \varphi(x):=\psi(x) \cdot(\sqrt{1+x}-1) .
$$

Then all the following hold:

(i) $\varphi$ is increasing, continuous on $[0, \infty)$, and $\lim _{x \rightarrow 0} \varphi(x)=0, \lim _{x \rightarrow \infty} \varphi(x)=1$; in addition, $\varphi$ is bijective;

(ii) $\psi$ is increasing on $[0,3)$ and decreasing on $(3, \infty)$, continuous on $[0, \infty)$, and

$$
\lim _{x \rightarrow 0} \psi(x)=\lim _{x \rightarrow \infty} \psi(x)=0
$$

(iii) $\varphi(x) \leq \psi(x)$ for every $x \in[0,3]$, and the equality holds for $x=0$ and $x=3$;

(iv) Let $\varepsilon \in(0, \infty]$ and $\delta \in(0, \infty)$.

1. Suppose that $\varepsilon \in\left(0,4^{-1}\right)$. Then the system of inequalities

$$
\varphi(x)>\delta, \quad \psi(x)<\varepsilon
$$

has (at least) a solution, if

$$
\sqrt{\delta}-\delta<\varepsilon \quad \text { and } \quad \delta<\varepsilon
$$

Conversely, if there exists a solution of the system (3.2) in $(0,3)$, then $\sqrt{\delta}-\delta<$ $\varepsilon$. 
2. Suppose that $\varepsilon>4^{-1}$. Then the system of inequalities has a solution if and only if $\delta \in(0,1)$.

In both cases, there exists $\mu>0$ such that every $x \in\left(\varphi^{-1}(\delta), \varphi^{-1}(\delta)+\mu\right)$ is a solution of the system 3.2 .

Proof. The first two assumption were discussed in [5, Remark 3.5] and is nothing but a straightforward computation that leads us to the desired conclusion. For (iii), firstly observe that $\varphi(0)=\psi(0)=0$ and $\varphi(3)=\psi(3)=4^{-1}$. Secondly, simply making some computations, for $x \in(0,3)$,

$$
\varphi(x) \leq \psi(x) \quad \Longleftrightarrow \quad \sqrt{1+x} \leq 2 .
$$

But the last inequality is obviously true.

The proof of the item (iv) is composed by two cases: $\varepsilon \in\left(0,4^{-1}\right)$ and $\varepsilon>4^{-1}$. Choose $\varepsilon \in\left(0,4^{-1}\right)$. Suppose that $\delta<\varepsilon$ and $\sqrt{\delta}-\delta<\varepsilon$. Since $\delta<\varepsilon \leq 4^{-1}$, we have that $\varphi^{-1}(\delta) \in(0,3)$. Using the properties of the functions $\varphi$ and $\psi$, we deduce that, for all $u \in\left(\varphi^{-1}(\delta), 3\right), \delta<\varphi(u)<\psi(u)$. Suppose that $\psi(u) \geq \varepsilon$, for all $u$, and note that this yields $\varphi(u)(\sqrt{1+u}-1)^{-1} \geq \varepsilon$. Passing to the limit with $u \rightarrow \varphi^{-1}(\delta)$ (using the continuity of $\varphi$ ), we deduce

$$
\frac{\delta}{\sqrt{1+\varphi^{-1}(\delta)}-1} \geq \varepsilon
$$

Denoting $\alpha:=1+\varphi^{-1}(\delta)$, it is convenient to observe that

$$
\frac{\delta}{\sqrt{1+\varphi^{-1}(\delta)}-1}=\frac{\delta}{\sqrt{\alpha}-1}=\sqrt{\delta}-\delta .
$$

This equality follows by using, in two different ways, the relation $\varphi\left(\varphi^{-1}(\delta)\right)=\delta$, i.e.,

$$
\left(\frac{1}{\sqrt{\alpha}}-\frac{1}{\alpha}\right)(\sqrt{\alpha}-1)=\delta \Longleftrightarrow \frac{(\sqrt{\alpha}-1)^{2}}{\alpha}=\delta .
$$

Indeed, on one hand we have that $\frac{\delta}{\sqrt{\alpha}-1}=\frac{1}{\sqrt{\alpha}}-\frac{1}{\alpha}$ and, on the other hand

$$
1-\frac{2}{\sqrt{\alpha}}+\frac{1}{\alpha}=\delta \quad \text { and } \quad \frac{\sqrt{\alpha}-1}{\sqrt{\alpha}}=\sqrt{\delta} .
$$

Subtracting the last two equality side by side we obtain relation $(3.3)$. So we have that $\sqrt{\delta}-$ $\delta \geq \varepsilon$, a contradiction. Thus, there exists $\bar{u} \in\left(\varphi^{-1}(\delta), 3\right)$ such that $\delta<\varphi(\bar{u})<\psi(\bar{u})<\varepsilon$. Moreover, we deduce that there exists a whole interval of solutions of the system (3.2), that is $\left(\varphi^{-1}(\delta), \varphi^{-1}(\delta)+\mu\right)$.

If the system $(3.2)$ admits a solution in $(0,3)$, then there exists $\bar{x} \in(0,3)$ such that we have $\delta<\varphi(\bar{x})<\psi(\bar{x})<\varepsilon$. Hence $\delta<4^{-1}$ and $\varphi^{-1}(\delta) \in(0, \bar{x})$. It follows that, for all 
$u \in\left(\varphi^{-1}(\delta), \bar{x}\right), \delta<\varphi(u)<\psi(u)<\varepsilon$. Passing to the limit with $u \rightarrow \varphi^{-1}(\delta)$, we obtain that

$$
\delta=\varphi\left(\varphi^{-1}(\delta)\right)=\psi\left(\varphi^{-1}(\delta)\right)\left(\sqrt{\varphi^{-1}(\delta)+1}-1\right)<\varepsilon\left(\sqrt{\varphi^{-1}(\delta)+1}-1\right) .
$$

Since $\sqrt{\varphi^{-1}(\delta)+1}-1 \in(0,1)$, we conclude that $\delta<\varepsilon$ and

$$
\frac{\delta}{\sqrt{\varphi^{-1}(\delta)+1}-1}=\sqrt{\delta}-\delta<\varepsilon .
$$

Finally choose $\varepsilon>4^{-1}$. If the system 3.2 has a solution then, using the fact that $\operatorname{Im} \varphi=[0,1)$, then one should have $\delta \in[0,1)$. If $\delta \in[0,1)$, then every $x \geq 0$ is a solution for $\psi(x)<\varepsilon$ and every $x>\varphi^{-1}(\delta)$ is a solution for $\varphi(x)>\delta$. Hence every $x \in\left(\varphi^{-1}(\delta), \infty\right)$ is a solution of the system (3.2) and the proof is complete.

Theorem 3.3. Let $X, Y$ be finite dimensional spaces, $\emptyset \neq L \subset S_{X}, u \in \operatorname{int} Q \cap S_{Q}$, and $F: X \rightrightarrows Y$ be a multifunction with $(\bar{x}, \bar{y}) \in \operatorname{Gr} F$. Fix $\delta>0$ and $\varepsilon>0$ such that the system (3.2) has a solution. Let $d>0$ be a solution. Suppose that

(i) $\operatorname{Gr} F \cap[D(\bar{x}, d) \times D(\bar{x}, d)]$ is closed;

(ii) for every $y^{*} \in Q^{+}$with $\left\langle y^{*}, u\right\rangle=1$, and for every $(x, y) \in \operatorname{Gr} F \cap[B(\bar{x}, d) \times B(\bar{y}, d)]$, $z^{*} \in 2 d B_{Y^{*}}, x^{*} \in \widehat{D}^{*} F(x, y)\left(y^{*}+z^{*}\right)$, there exists $w \in L$ such that

$$
-\left\langle x^{*}, w\right\rangle \geq d\left\|y^{*}+z^{*}\right\|
$$

Then

$$
B(\bar{y}, \delta) \cap[\bar{y}-\operatorname{cone}\{u\}] \subset \widetilde{F}(B(\bar{x}, \varepsilon) \cap[\bar{x}+\text { cone } L]) .
$$

Proof. The hypotheses (i) and (ii), together with our fixed framework, allow us to obtain the conclusion of Theorem 3.10 from [9], that is, for every $a \in(0, d)$, there exists $\theta>0$ such that, for every $\rho \in(0, \theta)$ and for all $(x, y) \in \operatorname{Gr} F \cap[B(\bar{x}, r) \times B(\bar{x}, r)]$,

$$
B(y, \rho a) \cap[y-\text { cone }\{u\}] \subset \widetilde{F}(B(\bar{x}, \rho) \cap[x+\text { cone } L])
$$

for some $r>0$. In particular, the above inclusion holds for $(\bar{x}, \bar{y}) \in \operatorname{Gr} F$.

Based upon the above inclusion and our assumptions, it suffices to prove that one can choose the constants $a$ and $\theta$ with the property $\theta<\varepsilon$ and $\delta<a \theta$.

Firstly, we take a brief look at the proof of Theorem 3.10 from [9], in order to note how the constants were fixed. Take

$$
a \in(0, d), \quad b \in\left((1+a)^{-1} a,(1+d)^{-1} d\right) \subset(0,1),
$$

and $\theta>0$ such that

$$
b^{-1} a \theta<2^{-1} r, \quad(1+a)^{-1} a<b+\theta<(1+d)^{-1} d .
$$


Secondly, we will use this inequality and some simple computation to find the desired constants. It is clear that $r=d$ and there exist $\mu, \nu>0$ such that

$$
b=(1+a)^{-1} a+\mu, \quad \theta=(1+d)^{-1} d-(1+a)^{-1} a-\mu-\nu .
$$

Hence $a \theta=a\left((1+d)^{-1} d-(1+a)^{-1} a-\mu-\nu\right)$ and the inequality $b^{-1} a \theta<2^{-1} d$ becomes

$$
a\left((1+d)^{-1} d-(1+a)^{-1} a-\mu-\nu\right)<2^{-1} d\left((1+a)^{-1} a+\mu\right) .
$$

The last inequality it is a simple consequence of the following one

$$
a\left((1+d)^{-1} d-(1+a)^{-1} a\right)<2^{-1} d(1+a)^{-1} a,
$$

which is equivalent with the inequality

$$
0<d^{2}-d+2 a
$$

Note that the constants $\mu$ and $\nu$ can be chosen arbitrarily small. In order to guarantee that $\delta<a \theta$ it is enough to ensure that

$$
\delta<a\left((1+d)^{-1} d-(1+a)^{-1} a\right)
$$

and for doing this we will take a close look at the function

$$
f:(0, d) \rightarrow \mathbb{R}, \quad f(a)=a\left((1+d)^{-1} d-(1+a)^{-1} a\right)
$$

A straightforward calculation gives us that the point $\bar{a}=\sqrt{1+d}-1$ is a maximum point and $f(\bar{a})=(\sqrt{1+d}-1)\left(\sqrt{1+d}^{-1}-(1+d)^{-1}\right)$.

As seen before, the point $\bar{a}$ is a good candidate for $a$ if, in addition, it satisfies the condition

$$
d^{2}-d+2 \sqrt{1+d}-2>0
$$

We show that this is indeed the case. For every positive $d$, we set $t:=\sqrt{1+d}$. Then $t>1$ and the above inequality becomes $t^{4}-3 t^{2}+2 t=t(t-1)(t+2)>0$ which is obviously true.

So, based on the above argument, hypothesis that $d$ is a solution of the system (3.2), we can choose $a=\bar{a}$ and

$$
\begin{aligned}
\theta & =(1+d)^{-1} d-(1+\bar{a})^{-1} \bar{a}-\mu-\nu<(1+d)^{-1} d-\sqrt{1+d}^{-1}(\sqrt{1+d}-1) \\
& <\sqrt{1+d}^{-1}-(1+d)^{-1} d<\varepsilon
\end{aligned}
$$

such that the desired inequality to hold. The proof is now complete. 
Theorem 3.4. Let $X, Y$ be finite dimensional spaces, $\emptyset \neq L \subset S_{X}, \emptyset \neq Q \subset Y$ a proper closed convex cone, $u \in \operatorname{int} Q \cap S_{Q}$, and $F: X \rightrightarrows Y$ be a multifunction with closed graph. Let $\varphi$ be the function introduced in Lemma 3.2. Fix $\varepsilon \in(0, \infty]$ and $\delta \in(0, \infty)$ such that one of the following set of conditions holds

(i) $\varepsilon>4^{-1}$ and $\delta \in[0,1)$;

(ii) $\delta<\varepsilon$ and $\sqrt{\delta}-\delta<\varepsilon$.

Suppose that $(\bar{x}, \bar{y}) \in \operatorname{Gr} F$ is an $(\varepsilon, \delta, u)$-approximate Pareto minimum point for the problem

$$
\min _{K} F(x) \text { subject to } x \in X \text {. }
$$

Moreover, suppose that $F$ has the Aubin property on $B(\bar{x}, 2 \delta) \times B(\bar{y}, 2 \delta)$. Then there exist $y^{*} \in Q^{+}$with $\left\langle y^{*}, u\right\rangle=1$, and $(x, y) \in \operatorname{Gr} F \cap\left[D\left(\bar{x}, \varphi^{-1}(\delta)\right) \times D\left(\bar{y}, \varphi^{-1}(\delta)\right)\right]$, $z^{*} \in$ $2 \varphi^{-1}(\delta) D_{Y^{*}}, x^{*} \in D^{*} F(x, y)\left(y^{*}+z^{*}\right)$, and for all $w \in L$ we have

$$
-\left\langle x^{*}, w\right\rangle \leq \varphi^{-1}(\delta)\left\|y^{*}+z^{*}\right\| .
$$

Proof. According to our assumptions on $\varepsilon$ and $\delta$, the system 3.2 has as solution at least an interval of points of the form $\left(\varphi^{-1}(\delta), \varphi^{-1}(\delta)+\mu\right)$, for some $\mu>0$.

Fixing $d \in\left(\varphi^{-1}(\delta), \varphi^{-1}(\delta)+\mu\right)$ and denoting $(\sqrt{1+d}-1)\left(\sqrt{1+d}^{-1}-(1+d)^{-1}\right)$ by $\rho$ which belongs to $[0,1)$, then $d=\varphi^{-1}(\rho)$. On the other hand

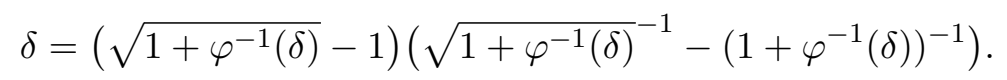

Consider now $\delta^{\prime \prime} \in(0,1)$ such that $\delta<\delta^{\prime \prime}<\rho$. Then

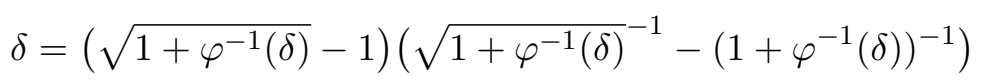

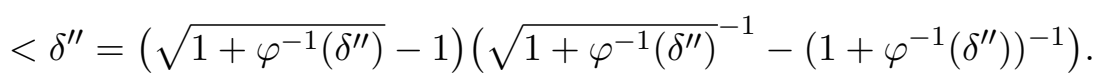

Now, for every such $\delta^{\prime \prime}$ close enough to $\delta$, then we still have

$$
{\sqrt{1+\varphi^{-1}\left(\delta^{\prime \prime}\right)}}^{-1}-\left(1+\varphi^{-1}\left(\delta^{\prime \prime}\right)\right)^{-1}<\varepsilon .
$$

So, for all $\delta^{\prime \prime}$ close enough to $\delta$, we have

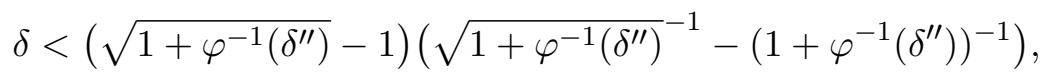

$$
\begin{aligned}
& {\sqrt{1+\varphi^{-1}\left(\delta^{\prime \prime}\right)}}^{-1}-\left(1+\varphi^{-1}\left(\delta^{\prime \prime}\right)\right)^{-1}<\varepsilon .
\end{aligned}
$$

Choose now $\left(\delta_{n}^{\prime \prime}\right) \subset(0,1)$ a sequence convergent towards $\delta$ such that $\delta_{n}^{\prime \prime}>\delta$, for all $n$, and consider another sequence $\left(\delta_{n}^{\prime}\right) \subset(0,1)$ such that $\delta<\delta_{n}^{\prime}<\delta_{n}^{\prime \prime}$, for all $n$. Notice that, in view of the above remarks, for $n$ large enough, one has

$$
\delta<\delta_{n}^{\prime}<\left(\sqrt{1+\varphi^{-1}\left(\delta_{n}^{\prime \prime}\right)}-1\right)\left({\sqrt{1+\varphi^{-1}\left(\delta_{n}^{\prime \prime}\right)}}^{-1}-\left(1+\varphi^{-1}\left(\delta_{n}^{\prime \prime}\right)\right)^{-1}\right),
$$


and

$$
{\sqrt{1+\varphi^{-1}\left(\delta_{n}^{\prime \prime}\right)}}^{-1}-\left(1+\varphi^{-1}\left(\delta_{n}^{\prime \prime}\right)\right)^{-1}<\varepsilon .
$$

By incompatibility result, Theorem 3.1, we deduce that

$$
B\left(\bar{y}, \delta_{n}^{\prime}\right) \cap[\bar{y}-\operatorname{cone}\{u\}] \not \subset \widetilde{F}(B(\bar{x}, \varepsilon) \cap[\bar{x}+\text { cone } L]),
$$

and it is the exact opposite of the conclusion of Theorem 3.3 applied for $\varepsilon, \delta_{n}^{\prime}$ and $\varphi^{-1}\left(\delta_{n}^{\prime \prime}\right)$. Or this means that one of the hypotheses of this theorem is not satisfied. Since the graph of $F$ is closed and based on the above inequalities, it is clear that the forth assumption is unfulfilled. Hence, for all $n$ large enough, there exist $y_{n}^{*} \in Q^{+}$with $\left\langle y_{n}^{*}, u\right\rangle=1$, and $\left(x_{n}, y_{n}\right) \in \operatorname{Gr} F \cap\left[B\left(\bar{x}, \varphi^{-1}\left(\delta_{n}^{\prime \prime}\right)\right) \times B\left(\bar{y}, \varphi^{-1}\left(\delta_{n}^{\prime \prime}\right)\right)\right]$, and $z_{n}^{*} \in 2 \varphi^{-1}\left(\delta_{n}^{\prime \prime}\right) B_{Y^{*}}, x_{n}^{*} \in$ $\widehat{D}^{*} F\left(x_{n}, y_{n}\right)\left(y_{n}^{*}+z_{n}^{*}\right)$ such that, for all $w \in L$,

$$
-\left\langle x_{n}^{*}, w\right\rangle \leq d_{\delta_{n}^{\prime \prime}}\left\|y_{n}^{*}+z_{n}^{*}\right\|
$$

Since $u \in \operatorname{int} Q$ and $y_{n}^{*} \in Q^{+}$with $\left\langle y_{n}^{*}, u\right\rangle=1$, for all $n$, by virtue of [10, Lemma 2.2.17], it follows that $\left(y_{n}^{*}\right)$ is bounded. It is clear that $\left(x_{n}, y_{n}\right)$ and $\left(z_{n}^{*}\right)$ are bounded. We deduce that $\left(y_{n}^{*}+z_{n}^{*}\right)$ is bounded and, since, for $n$ large enough, $\left(x_{n}, y_{n}\right) \in B(\bar{x}, 2 \delta) \times B(\bar{y}, 2 \delta)$, by means of Aubin property of $F$ we assumed, we deduce that $\left(x_{n}^{*}\right)$ is bounded too. Since all spaces are finite dimensional and the involved sequences are bounded, we can find a common sequence on which they are convergent. Without relabeling, we can write: $x_{n}^{*} \in x^{*}, y_{n}^{*} \rightarrow y^{*} \in Q^{+}$with $\left\langle y^{*}, u\right\rangle=1, z_{n}^{*} \rightarrow z^{*} \in 2 \varphi^{-1}(\delta) D_{Y^{*}}, x_{n} \rightarrow x \in D\left(\bar{x}, \varphi^{-1}(\delta)\right)$, $y_{n} \rightarrow y \in D\left(\bar{y}, \varphi^{-1}(\delta)\right)$. Using the closedness of the graph of $F$ and the definition of the limiting coderivative, we get $(x, y) \in \operatorname{Gr} F, x^{*} \in D^{*} F(x, y)\left(y^{*}+z^{*}\right)$. Moreover, for all $w \in L$, we get

$$
-\left\langle x^{*}, w\right\rangle \leq \varphi^{-1}(\delta)\left\|y^{*}+z^{*}\right\|
$$

The proof is complete.

Example 3.5. Let $X=\mathbb{R}, Y=\mathbb{R}^{2}, L=\{1\}, Q=\mathbb{R}_{+}^{2}, u=2^{-1 / 2}(1,1) \in \operatorname{int} Q \cap S_{Q}$, and consider the function

$$
f: X \rightarrow Y, \quad f(x)=\left(-\frac{x}{2},-x-x^{3}\right) .
$$

Then $(0,(0,0))$ is an $(\varepsilon, \delta, u)$-aproximate Pareto minimum point for $f$ with respect to $L$, if $0<\delta<4^{-1}$ and $\varepsilon \in\left[4^{-1}, x_{0}\right)$, where $x_{0} \in[0,1)$ is the unique solution of equation $x^{3}+x=2^{3 / 2} \delta$. Moreover, for such $(\varepsilon, \delta)$ pairs, one has that

$$
\delta<\varepsilon \quad \text { and } \quad \sqrt{\delta}-\delta<\varepsilon
$$

Additionally the graph of $f$ is closed, and $f$ has the Aubin property on $B(0,2 \delta) \times$ $B((0,0), 2 \delta)$. Using the calculus rules for the normal coderivative (see 12 , Theorems 1.38 
and 1.90]) we have that, for any $x \in X$ and $y^{*}=(\alpha, \beta) \in Y^{*}$,

$$
D^{*} f(x, f(x))\left(y^{*}\right)=-\frac{1}{2}\left(\alpha+2 \beta+6 \beta x^{2}\right) .
$$

Choosing $y^{*}=\sqrt{2}(1,1) \in Q^{+}$we have $\left\langle y^{*}, u\right\rangle=1$. Then, letting $z^{*}=0$ and $x^{*} \in$ $D^{*} f(x, f(x))\left(y^{*}\right)$ we have that, for $w=1$,

$$
-\left\langle x^{*}, w\right\rangle=-\frac{9}{2}<\varphi^{-1}(\delta)\left\|y^{*}+z^{*}\right\|
$$

Whence the conclusion of Theorem 3.4 holds.

\section{Concluding remarks}

In many practical problems from optimal control, shape optimization, dynamic systems, parameters play an important role especially when someone tries to use computational approaches in order to get at least an approximate solution. An important task that rise a lot of issues consists of tuning the parameters.

In this paper it was studied the class of vector optimization problem with geometric constraints from two point of views: the possibility of obtaining some Clarke's penalization type results and the possibility of finding some optimality conditions. Besides considering the directional setting, the minimum concept studied in this paper contains two constants that, in some sense, measure the approximate character of efficiency. By this paper, we had aimed to prove that some results from the papers [2, 3, 5] still hold for approximate directional Pareto minima. The novelty here is the fact that it's required a careful tracking (see Lemma 3.2) of the aforementioned constants that are involved in the definition of the minimality concepts.

The next possible step that one could do in the directional efficiency area is to consider some classical algorithms (proximal point type algorithm, fixed point algorithm for solving generalized equations) and to study the impact of directional framework in the convergence of the algorithms, a priori/posteriori estimations etc. For this reason, we consider the concepts of approximate minima of great interest for future researches.

\section{Acknowledgments}

This research was supported by the European Social Fund in the framework of The Human Capital Operational Program 2014-2020, POCU/380/6/13/123623, "Doctoral students and postdoctoral researchers ready for the labor market". 


\section{References}

[1] T. Q. Bao and B. S. Mordukhovich, Relative Pareto minimizers for multiobjective problems: Existence and optimality conditions, Math. Program. 122 (2010), no. 2, Ser. A, 301-347.

[2] T. Chelmuş and M. Durea, Exact penalization and optimality conditions for constrained directional Pareto efficiency, Pure Appl. Funct. Anal. 5 (2020), no. 3, 533553.

[3] T. Chelmus, M. Durea and E.-A. Florea, Directional Pareto efficiency: Concepts and optimality conditions, J. Optim. Theory Appl. 182 (2019), no. 1, 336-365.

[4] A. L. Dontchev and R. T. Rockafellar, Implicit Functions and Solution Mappings: A view from variational analysis, Second edition, Springer Series in Operations Research and Financial Engineering, Springer, Berlin, 2014.

[5] M. Durea, Optimality conditions for approximate Pareto minimality, Numer. Funct. Anal. Optim. 41 (2020), no. 8, 883-900.

[6] M. Durea, J. Dutta and C. Tammer, Lagrange multipliers for $\varepsilon$-Pareto solutions in vector optimization with nonsolid cones in Banach spaces, J. Optim. Theory Appl. 145 (2010), no. 1, 196-211.

[7] M. Durea, M. Panţiruc and R. Strugariu, Minimal time function with respect to a set of directions: Basic properties and applications, Optim. Methods Softw. 31 (2016), no. $3,535-561$.

[8] _ A new type of directional regularity for mappings and applications to optimization, SIAM J. Optim. 27 (2017), no. 2, 1204-1229.

[9] E.-A. Florea and D. Maxim, Directional openness for epigraphical mappings and optimality conditions for directional efficiency, Optimization 70 (2021), no. 2, 321-344.

[10] A. Göpfert, H. Riahi, C. Tammer and C. Zălinescu, Variational Methods in Partially Ordered Spaces, CMS Books in Mathematics/Ouvrages de Mathématiques de la SMC 17, Springer-Verlag, Berlin, 2003.

[11] D. S. Kim and T. Q. Son, An approach to $\varepsilon$-duality theorems for nonconvex semiinfinite multiobjective optimization problems, Taiwanese J. Math. 22 (2018), no. 5, 1261-1287. 
[12] B. S. Mordukhovich, Variational Analysis and Generalized Differentiation I: Basic theory, Grundlehren der Mathematischen Wissenschaften 330, Springer-Verlag, New York, 2006.

[13] Q. K. Phan and M. T. Nguyen, Existence and boundedness of second-order KarushKuhn-Tucker multipliers for set-valued optimization with variable ordering structures, Taiwanese J. Math. 22 (2018), no. 4, 1001-1029.

[14] J. J. Ye, The exact penalty principle, Nonlinear Anal. 75 (2012), no. 3, 1642-1654.

[15] J. J. Ye and X. Y. Ye, Necessary optimality conditions for optimization problems with variational inequality constraints, Math. Oper. Res. 22 (1997), no. 4, 977-997.

[16] A. J. Zaslavski, Stability of exact penalty for nonconvex inequality-constrained minimization problems, Taiwanese J. Math. 14 (2010), no. 1, 1-19.

Teodor Chelmuş

Faculty of Mathematics, Alexandru Ioan Cuza University, Bd. Carol I, nr. 11, 700506 Iaşi, Romania

E-mail address: teo1chelmus@gmail.com 